\title{
G45R mutation in the nonstructural protein 1 of A/Puerto Rico/8/1934 (H1N1) enhances viral replication independent of dsRNA- binding activity and type I interferon biology
}

\author{
Challika Kaewborisuth ${ }^{1,2}$, Mark Zanin², Hans Häcker ${ }^{3}$, Richard J. Webby ${ }^{2}$ and Porntippa Lekcharoensuk ${ }^{1,45^{*}}$
}

\begin{abstract}
Background: The nonstructural protein 1 (NS1) of influenza A viruses can act as a viral replication enhancer by antagonizing type I interferon (IFN) induction and response in infected cells. We previously reported that A/Puerto Rico/8/1934 (H1N1) (PR8) containing the NS1 gene derived from A/swine/IA/15/1930 (H1N1) (IA30) replicated more efficiently than the wild type virus. Here, we identified amino acids in NS1 critical for enhancing viral replication.

Methods: To identify a key amino acid in NS1 which can increase the virus replication, growth kinetics of PR8 viruses encoding single mutation in NS1 were compared in A549 cells. NS1 mutant functions were studied using dsRNA-protein pull down, RIG-I mediated IFN $\beta$-promoter activity assays and growth curve analysis in murine lung epithelial type I (Let1) cells.

Results: The G45R mutation in the NS1 of PR8 (G45R/NS1) virus is critical for the enhanced viral replication in A549 cells. G45R/NS1 slightly decreased NS1 binding to dsRNA but did not interfere with its suppression of RIG-Imediated type I IFN production. Likewise, replication of G45R/NS1 virus was increased in comparison to wild type virus in both wild type and type I interferon receptor null Let1 cells.

Conclusions: The non-conserved amino acid, R45, enhances viral replication which is apparently independent of dsRNA binding and suppression of type I IFN, suggesting a non-characterized function of NS1 for the enhanced viral replication. As G45R/NS1 virus induced the type I IFN induction and response in infected A549 cells, it is also interesting to investigate virus virulence for further studies.
\end{abstract}

Keywords: Influenza A virus, NS1, G45R, dsRNA binding, Type I IFN, Virus replication

\section{Background}

Influenza A virus has evolved a variety of mechanisms to evade host cell defenses to facilitate efficient transmission [1, 2], replication and virulence [3]. Escape from host innate immune responses allows influenza A virus to proficiently replicate in the cells. The most important

\footnotetext{
* Correspondence: fvetptn@ku.ac.th

${ }^{1}$ Interdisciplinary Graduate Program in Genetic Engineering, The Graduate School, Kasetsart University, Bangkok 10900, Thailand

${ }^{4}$ Department of Microbiology and Immunology, Faculty of Veterinary

Medicine, Kasetsart University, 50th Ngamwongwan Rd., Chatuchak, Bangkok 10900, Thailand

Full list of author information is available at the end of the article
}

innate immune response against influenza $\mathrm{A}$ virus is the interferon (IFN) system [4]. There are three types of IFN families including type I $(-\alpha,-\beta,-\omega$ and $-\varepsilon)$, type II $(-\gamma)$ and type III $(-\lambda s$ or IL28/29) [5]. Type I IFNs, especially $-\alpha / \beta$, are essential for host defense against viral infection. It is induced following the recognition of viral components or RNA by pathogen recognition receptors (PRRs) such as retinoic acid-inducible gene I (RIG-I) in the cytoplasm and toll-like receptors (TLR) in the endosome and on the cell surface [6-8].

IFN signaling is mediated via binding between IFN and its receptor, which triggers downstream signaling via 
Janus Activated Kinase (JAK) and Tyrosine Kinae-2 (TYK2) followed by Signal Transducer and Activators of Transcription (STAT) proteins [9]. After activation, STATs translocate into the nucleus to initiate transcription of IFN-stimulated genes (ISGs), such as 2'-5'oligoadenylate synthetase (OAS1), dsRNA-dependent protein kinase $\mathrm{R}$ (PKR) and Mx1, which have antiviral functions $[10,11]$.

The non-structural protein 1 (NS1) of influenza A virus plays an important role in counteracting the IFN $\alpha$ / $\beta$ system [12], thereby preventing the generation of an antiviral state in the cell and facilitating efficient viral replication [13, 14]. This action of NS1 was shown at multiple levels in vitro and is different among influenza A virus strains [15]. NS1 contains two major functional domains, an N-terminal RNA-binding domain (RBD), which binds dsRNA, and a C-terminal effector domain (ED). The RBD has been proposed to sequester dsRNA from RIG-I [16] and dsRNA-activated antiviral enzymes such as OAS1 $[17]$ and PKR $[18,19]$ to prevent their antiviral activities.

Previously, we reported that reassortant viruses containing internal gene segments derived from the A/swine/IA/ 15/30 H1N1 virus (IA30) with the hemagglutinin (HA) and neuraminidase (NA) from the avian influenza virus $\mathrm{H} 5 \mathrm{~N} 1$ or swine influenza virus H3N2 replicated more efficiently than those with internal gene segments from A/ Puerto Pico/8/1934 (H1N1) (PR8) in Madin Darby canine kidney (MDCK) and Vero cells [20]. Among the IA30 genes, IA30 NS has been shown to increase PR8 virus replication more efficiently than wild type PR8 NS1 [21]. In this report, we have identified a non-conserved residue of NS1 that enhanced viral replication independent of its dsRNA-binding ability and the IFN antagonist function.

\section{Results}

\section{The RNA-binding domain of IA30 NS1 increased virus} replication

We reported previously that IA30 replicated to higher titers than PR8 [20] and it was mediated by the IA30 NS [21]. In this study, we compared the growth kinetics of viruses generated by reverse genetics based on X31 containing the $\mathrm{HA}$ and $\mathrm{NA}$ of A/Hong Kong/1/1968 (H3N2), the PB2, PB1, PA, NP, and M of PR8 and the NS of either PR8 or IA30. The virus containing IA30 NS grew to significantly higher titers at 48 and $72 \mathrm{~h}$ post infection (hpi) than the virus containing the PR8 NS (Fig. 1b). To identify the IA30 NS1 domain responsible for increasing viral replication, we generated viruses containing chimeric NS genes. Chimera 1 contained the RBD derived from PR8 NS1 and the ED derived from IA30 NS1 whilst Chimera 2 contained the RBD derived from IA30 NS1 and the ED derived from PR8 (Fig. 1a). Chimera 2 reached significantly higher titers compared to PR8 NS1 virus, whilst Chimera 1 had a comparable replication rate to the PR8 NS1 virus (Fig. 1b). These results indicated that the IA30 RBD was involved in increasing viral replication.

We performed multiple alignments of the deduced amino acids of both chimeras. The result shows that few amino acids on NS2 of both chimeras are different. NS2 has a nuclear export signal and interacts with $\mathrm{M} 1$ via its C-terminal domain (aa 81-100) to transport the vRNP from nucleus to cytoplasm for viral assembly (Shimizu et al., 2011) and also regulates the accumulation of viral RNA species including viral RNA, cRNA and mRNA, which are important for viral transcription and replication (Robb et al., 2009). According to NS2 functions, we cannot exclude that NS2 translated from chimeric 1 and 2 genes may have an effect on the replication.

\section{A single amino acid substitution, G45R, in PR8 NS1 contributed to enhanced viral replication}

To determine amino acids in the RBD of IA30 NS1 that contributed to increased viral replication, amino acid sequences of PR8 and IA30 NS1s were compared. Eight residues in the RBD were different between the two strains. We selected four point mutations at positions 45, 55, 68 and 70, which did not affect NS2 expression, to introduce into the RBD coding region of PR8 NS1 to generate viruses containing PR8 NS1 with G45R, K55E, I68V or K70E mutations via reverse genetics [22]. Growth kinetics of the viruses was assessed in A549 cells at a multiplicity of infection (MOI) of 0.01. Viruses with NS1 mutations at G45R and K55E (G45R and K55E/NS1s) grew to higher titers compared to the wild type (WT), $\mathrm{I} 68 \mathrm{~V}$ and $\mathrm{K} 70 \mathrm{E} / \mathrm{NS} 1 \mathrm{~s}$ viruses. The G45R/NS1 virus replicated to the highest titer $(P \leq 0.001)$ at 48 to 96 hpi while the replication rates of $168 \mathrm{~V}$ and $\mathrm{K} 70 \mathrm{E} / \mathrm{NS} 1 \mathrm{~s}$ mutants were similar to the WT/NS1 virus (Fig. 1b). The increased virus replication with K55E substitution in PR8 NS1 has been addressed previously for cell-based vaccine production [23]. As the G45R/NS1 virus replicated to the highest titer, it was selected for further studies.

\section{G45R/NS1 did not increase binding to dsRNA in vitro}

The RBD of NS1 binds to dsRNA to counteract the host IFN system and facilitate virus replication $[16,17]$. As viruses containing G45R/NS1 replicated at the highest rate, we sought to elucidate its impact on RNA binding activity in vitro. To assess the dsRNA binding ability of the G45R/ NS1 protein, the RBD of PR8 NS1 [WT, G45R and the dsRNA binding deficient mutant R38AK41A (AA)] [13] were expressed in E. coli. The expressed proteins were purified by affinity chromatography and used in the dsRNA-protein pull down assay. WT/NS1 bound to dsRNA with high affinity while the deficient mutant, AA/NS1, failed to bind dsRNA (Fig. 2). However, G45R/ NS1 did not increase the binding ability of NS1 compared to WT/NS1 (Fig. 2). All three replicates of the pull down reactions performed separately yielded similar results 


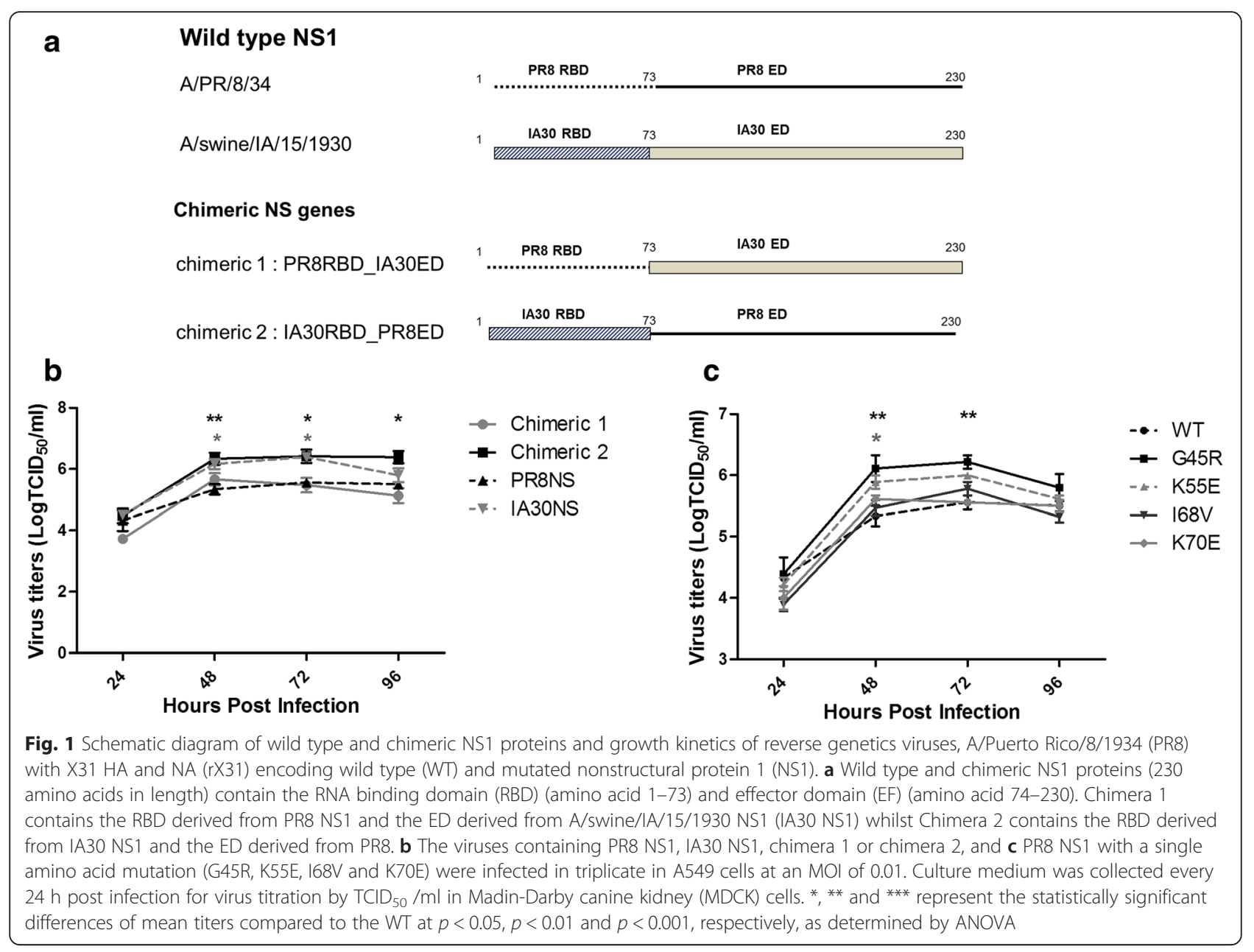

(Additional file 1: Fig. S1). Therefore, G45R/NS1 does not play a significant role in the dsRNA-NS1 interaction in vitro.

\section{G45R/NS1 did not increase inhibition of RIG-I mediated IFN $\beta$-promoter activation}

RIG-I is a cytoplasmic pathogen sensor that is crucial to recognize viral RNA, particularly 5'-ppp ssRNA and dsRNA, and trigger type I IFN induction to combat viral infection $[24,25]$. NS1 has been shown to inhibit RIG-I-

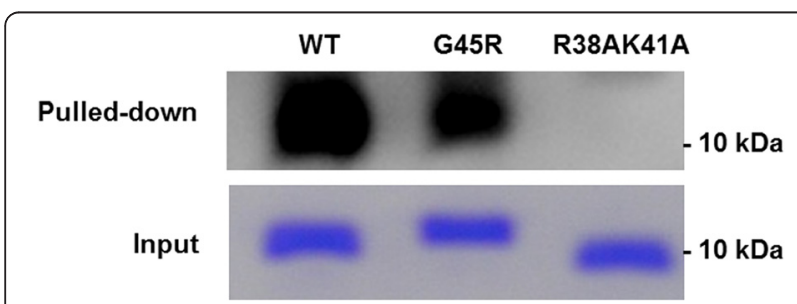

Fig. 2 dsRNA-RBD NS1 pull down assay. The G45R/NS1 bound to dsRNA with lower affinity than the wild type (WT). The R38AK41A as a negative control failed to bind with dsRNA. The input NS1_RBDs for dsRNA-protein pulled down assay were loaded and run onto $15 \%$ acrylamide gel. The input proteins were detected by Coomassie brilliant blue staining mediated IFN induction by forming a complex with RIG-I partly via the RNA-binding domain [26]. We assessed whether G45R/NS1 was able to inhibit RIG-I mediated IFN synthesis in $293 \mathrm{~T}$ cells co-transfected with plasmids including pCAGGS_PR8NS1 (WT, G45R or AA) or empty pCAGGS as a control, pRIG-I and pfirefly luciferase driven by the IFN $\beta$ promoter and $\mathrm{p} R e$ nilla Luciferase. RIG-I mediated IFN $\beta$-promoter activation was measured by dual luciferase reporter assay. The IFN $\beta$ promoter was strongly activated in cells transfected with RIG-I; however, in the presence of NS1, IFN $\beta$ activation was reduced. AA/NS1 was less efficient in suppressing RIG-I-mediated IFN $\beta$-promoter activity, as expected (Fig. 3). The inhibition of RIG-I mediated IFN $\beta$ promoter activity by WT and G45R/NS1 proteins were similar while the NS1 expressions by each virus in the transfected cells were not different (Additional file 2: Fig. S2). This confirmed that the different luciferase signals did not come from different levels of NS1 expression. Therefore, the increased replication of G45R/NS1 virus was not due to NS1-mediated alterations of RIG-I mediated IFN $\beta$ promoter activation. 


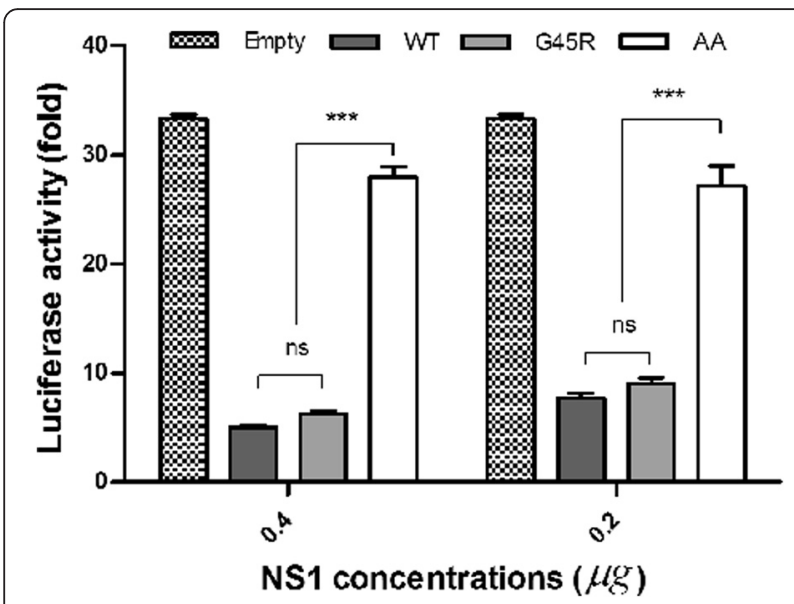

Fig. 3 RIG-I mediated IFN $\beta$-promoter activity in 293 T cells expressing PR8 NS1 (wild type; WT, G45R and R38AK41A; AA), RIG I and IFNßpromoter luciferase reporter at $24 \mathrm{~h}$ post infection. WT and G45R NS1s decreased the luciferase expression in dose-dependent manner by inhibition of RIG-I mediated IFNß-promoter activity. The double mutant R38AK41A served as a negative control failed to inhibit RIG I mediated luciferase expression via IFN $\beta$-promoter. ${ }^{* *}$ represents the statistically significant difference of mean luciferase activity compared to the WT and G45R at $p<0.001$, as determined by ANOVA

\section{G45R/NS1 increased virus replication independent of type I IFN induction}

Our data suggested that the anti-RIG-I activity of the G45R/NS1 variant was similar to WT NS1. To test this hypothesis, we infected A549 cells with WT and G45R/ NS1 viruses at an MOI of 1 to measure type I IFN mRNA expression and single step growth kinetics. As shown earlier, the G45R/NS1 virus replicated more efficiently than the WT virus, which is particularly apparent early in infection. Interestingly, the G45R/NS1 virus induced greater expression of IFN $\alpha / \beta$ mRNA compared to the WT/NS1 (Fig. 4). As such, G45R/NS1 did not suppress type I IFN expression to facilitate virus replication.

Based on these observations we concluded that G45R/ NS1 does not possess type I IFN suppressive activity and hence is not responsible for the increased G45R/NS1 virus replication. To further evaluate the relevance of type I IFNs for virus replication, we conducted growth kinetics experiments using WT, G45R and AA/NS1s viruses in WT and type I interferon receptor null (IFNAR${ }^{n u l l}$ ) murine lung epithelial type I (Let1) cells [27]. If type I IFNs were involved in the difference between the replication kinetics of WT and G45R/NS1 viruses, replication of the G45R/NS1 virus should be greater than the WT/ NS1 virus in WT Let1 cells yet similar in IFNAR ${ }^{\text {null }}$ Let1 cells. As shown in Fig. 5, the G45R/NS1 virus replicated to higher titers compared to the WT/NS1 virus in both WT and IFNAR ${ }^{\text {null }}$ Let1 cells at every time point investigated, further supporting our conclusion that modulation of type I interferon biology, at the level of RIG-I- induced IFN activation or at later levels of type I interferon activity, is not the reason for the increased replicative capacity of the G45R/NS1 virus observed.

\section{Discussion}

The NS1 protein of influenza A virus is multifunctional and associated with increased viral pathogenicity and replication by counteracting host antiviral defense mechanisms [28]. NS1 inhibits IFN $\alpha / \beta$ induction and the antiviral response through different pathways $[4,15,29-31]$, though some functions are strain-specific [32, 33]. It was shown previously that recombinant viruses with different HA and NA subtypes in an IA30 virus background had much higher replication rates compared to viruses in a PR8 virus background [20]. Recently, IA30 NS has been shown to involve in the increased virus replication [21]. Here, we investigated the roles of amino acids in IA30 NS1 in enhancing viral replication. We constructed viruses using reverse genetics that contained the HA and NA genes from X31 in a PR8 background and demonstrated that the single amino acids substitution, G45R, in the RBD of PR8 NS1 accelerated viral replication.

The RBD of NS1 plays a major role in inhibiting host defense mechanisms, in particular type I IFN production. It may sequester dsRNA from PRRs and ISG products that require activation by dsRNA, such as RIG-I, PKR and OAS1 $[4,17,30]$. In addition, NS1 also interacts with these proteins to block their functions [19, 24]. Viruses containing the R38A mutation in NS1 were attenuated, lacked dsRNA binding and were sensitive to IFN $\beta$ [23]. However, replication of viruses containing the R38A NS1 mutation was improved in RNaseL knockout cells, suggesting that NS1 was involved in the inhibition of the 2'-5' OAS/RNaseL pathway, possibly by sequestering dsRNA and preventing activation of OAS1 [23].

Previously, in silico analysis suggested that the R45 on NS1 of the pandemic virus A/Texas/15/2009 (H1N1) increased the stability of the dsRNA-NS1 complex, which contributed to viral pathogenicity [34]. We investigated whether the enhanced viral replication mediated by G45R/NS1 was due to the increased dsRNA binding in vitro using dsRNA-NS1 pull-down assays. In these assays, G45R/NS1 did not show increased dsRNA-binding compared to WT/NS1. As G45R/NS1 did not act as predicted [34], we speculated that substitution of G45R on PR8 NS1 may impact dsRNA binding by steric hindrance. Nonetheless, NS1 functions do not depend solely on its dsRNA-binding activity. WSN-NS1 with the triple mutations R38A, K41A and S42G could not bind to dsRNA similar to R38A and $\mathrm{K} 41 \mathrm{~A}$, though the virus containing R38A, K41A and S42G decreased activation of the IFN $\beta$ promoter and had a higher replication rate compared to the virus containing R38A and K41A [13]. 


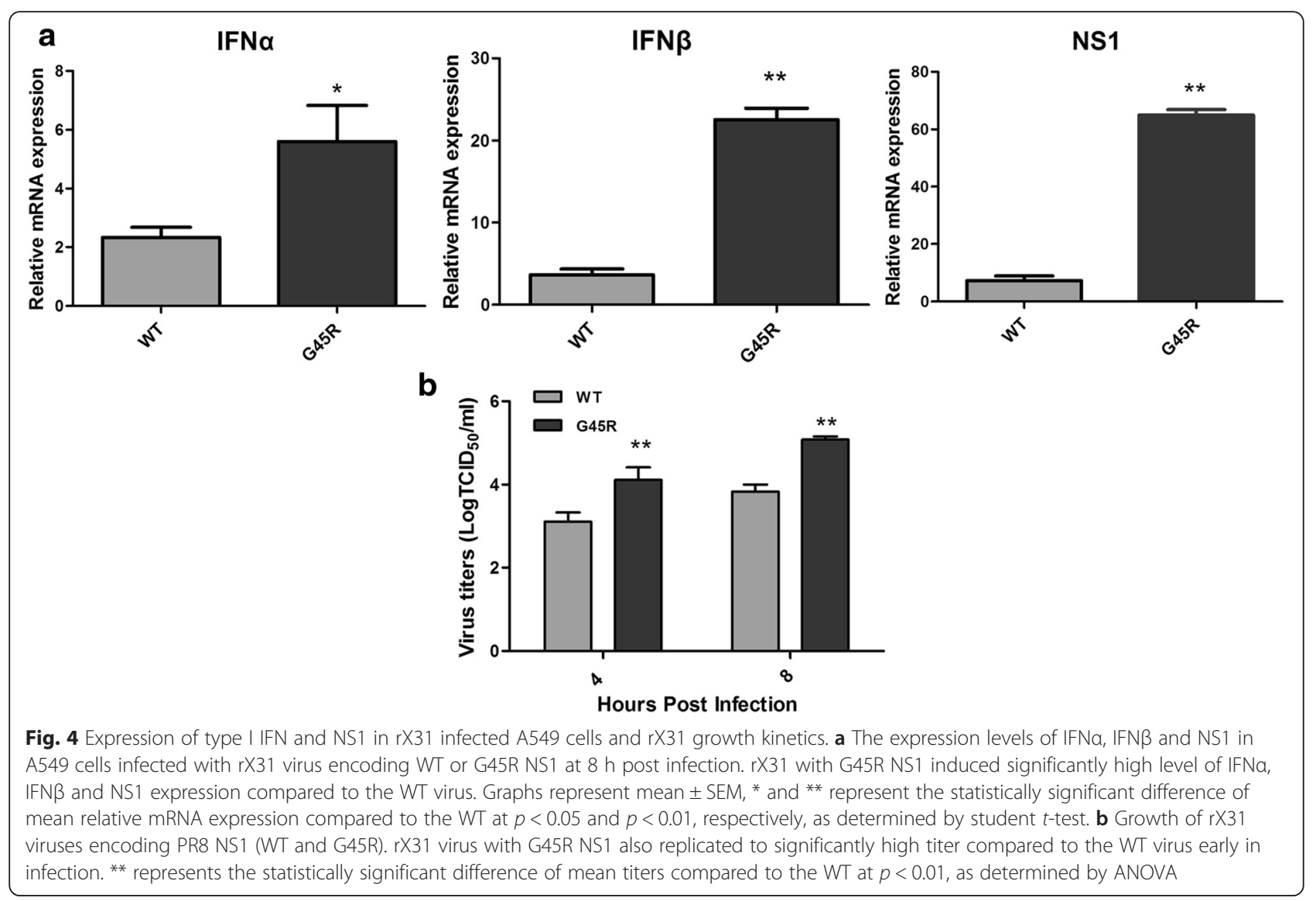

RIG-I is a crucial cytoplasmic sensor for dsRNA and 5'ppp-ssRNA that triggers downstream signaling to activate type I IFN production during virus infection [24, 25]. It has been reported that NS1 interferes with the RIG-I mediated IFN pathway partly through its RBD, increasing viral replication and pathogenicity $[35,36]$. We investigated whether G45R/NS1 increased virus replication by inhibiting RIG-I mediated IFN $\beta$-promoter activation. The reporter assay showed that G45R/NS1 suppressed the activation of IFN $\beta$-promoter comparable to WT/ NS1 (Fig. 3). We suggest that the G45R mutation in PR8 NS1 facilitated virus replication independent of

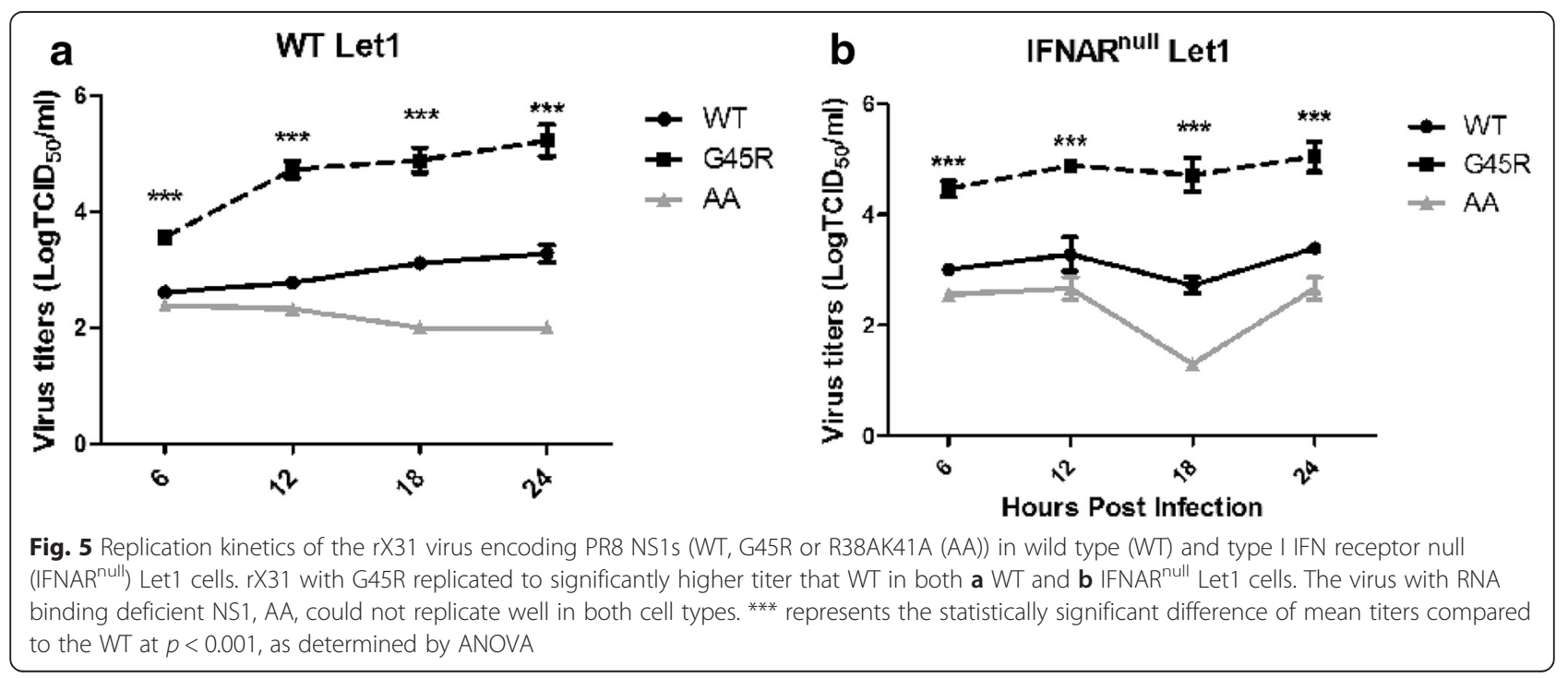


dsRNA-binding and RIG-I mediated IFN $\beta$ promoter activation.

We confirmed that type I IFNs induction was not relevant to G45R/NS1 virus replication by using IFNAR ${ }^{\text {null }}$ Let1 cells. G45R/NS1 replicated to higher titers compared to WT virus while AA/NS1 virus was attenuated in both WT and IFNAR ${ }^{\text {null }}$ Let1 cells (Fig. 5) suggesting that G45R/NS1 strongly influenced virus replication in a type I IFN induction-independent manner. In fact, high replication of G45R/NS1 was accompanied by increased type I IFN and STAT1 phosphorylation (Fig. 4 and Additional file 3: Fig. S3), conferring a strong activation of type I IFN signaling. Elevation of type I IFN expression can up-regulate the expression of various cytokines and chemokines to recruit immune cells to the site of infection. In addition, IFN $\alpha$ has been reported to enhance CCL5 and CCL10 expression in influenza virusinfected A549 cells [37]. Rapid virus replication, systemic spread and activation of IFN $\alpha / \beta$ signaling by influenza virus infection could lead to excessive production of cytokines and chemokines, resulting in increased morbidity and mortality in mice $[38,39]$. An adaptation of amino acids on NS1, F103L and M106I, of human influenza A/Hong Kong/1/68(H3N2) has been reported to be associated with the increased virus replication and virulence in mice and human [40] thereby the occurrence of G45R mutation on NS1 of pandemic human 2009 H1N1 virus might be one of crucial factors to cause severe disease. Even though G45R in NS1 presents in a minor population of the pandemic H1N1 2009 viruses, it has been proposed that G45R mutation might be related to the increased cytokine production observed during infections by these viruses [41].

\section{Conclusions}

We elucidated the function of the non-conserved amino acid, R45, in NS1, which facilitated viral replication by an undefined mechanism. G45R/NS1 does not increase the efficiency of binding to dsRNA or suppress IFN $\beta$ induction, which are the established functions of NS1. Thus, increased viral replication of G45R/NS is independent of dsRNA binding and type I IFN induction, suggesting that a non-characterized function of NS1 was responsible for the enhanced viral replication observed in this study. As we have demonstrated that G45R/NS1 virus induced the type I IFN induction and response in infected A549 cells, it is also interesting to investigate virus virulence for further studies.

\section{Methods}

\section{Cells lines}

Human embryonic kidney (293 T), human lung carcinoma (A549) and Mardin-Darby canine kidney (MDCK) cells were cultured in Opti-MEM I reduced serum medium (Gibco, Invitrogen), Kaighn's Modification of Ham's F-12 Medium (ATCC) or minimum essential media (Invitrogen), respectively, supplemented with $5 \%$ fetal bovine serum (Invitrogen), L-glutamine (Invitrogen), penicillin and streptomycin (Invitrogen). Murine lung epithelial type I cell line (Let1; wild type or type I IFN receptor null, IFNAR $\left.{ }^{\text {null }}\right)$, kindly provided by Alan Aderem and Alan Diercks [27], was cultured in Dulbecco's Modified Eagle Medium (Invitrogen) supplemented with $10 \%$ fetal bovine serum, penicillin and streptomycin. All cells were incubated in a humidified atmosphere of $5 \% \mathrm{CO}_{2}$ at $37^{\circ} \mathrm{C}$.

\section{Construction of plasmids}

Two chimeric NS genes-chimera 1 and chimera 2-comprising the 3'UTR and RBD of PR8 NS1 (amino acid 1-73) and the effector domain (ED) (amino acid 74-230) and 5' UTR derived from IA30 NS1 or vice versa were amplified from plasmids containing fulllength NS genes of both strains [20] using a fusion PCR technique with a pair of specific primers. This strategy was designed to keep the NS2 transcripts intact. To make a single point mutation on NS1 protein of PR8, the NS1 sequences of PR8 and IA30 were compared to determine the different amino acids between the two strains. Four different amino acids on RBD were selected and a single point mutation was introduced into PR8 NS gene using specific primers. Amino acids at position 45, 55, 68 or 70 of PR8 NS1 were replaced with those of IA30. The mutations did not interfere with NS2 expression as they are spliced out to generate the mature mRNA. The dsRNA-binding deficient NS1, R38AK41A (AA) was constructed using similar strategies. The mutated NS genes were digested and inserted into the bidirectional vector pHW2000 [22].

The RBD (amino acids 1-73) of PR8 NS1 genes were cloned into the $\mathrm{pQE} 80 \mathrm{~L}$ vector (Qiagen) for protein expression in E. coli strain BL21. The DNA sequences in the positive clones were verified by DNA sequencing (Macrogen, Korea).

\section{Generation of viruses by reverse genetics}

The viruses used in this study were generated by transfection using the eight-plasmid reverse genetics system in $293 \mathrm{~T}$ and MDCK cells as described previously [20, 42]. The plasmids used were as follows; NS1 from PR8 (WT or mutated PR8 NSs; G45R, K55E, I68V, K70E and AA), HA and NA from X31 or A/Hong Kong/1/1968 (H3N2) and the remaining five genes were from PR8. Briefly, $0.5 \mu \mathrm{g}$ of each of the eight plasmids were incubated with $12 \mu \mathrm{l}$ of X-tremeGENE9 transfection reagent (Roche) in $200 \mu \mathrm{l}$ reaction for $15 \mathrm{~min}$ before they were gently overlaid onto a 50:50 293 T-MDCK cell mixture. The transfected cells were cultured in Opti-MEM I 
reduced serum (Gibco, Invitrogen) containing $1 \mu \mathrm{g} / \mathrm{ml}$ of TPCK-treated trypsin and incubated at $37{ }^{\circ} \mathrm{C}$ with $5 \% \mathrm{CO}_{2}$. The reverse genetics viruses were recovered by collecting supernatant at $48-72 \mathrm{hpi}$, which was then used to inoculate the allantoic cavities of 9-day-old specific pathogen free (SPF) embryonated chicken eggs. The existence of the mutation on each NS1 was confirmed by sequencing.

\section{Virus infection}

The rescued viruses with different PR8 NS1 (WT, G45R or AA/NS1s) were used to infect A549 cells grown in 6well plates in triplicate wells at an MOI of 0.01 or 2 . The infected cells were maintained in Kaighn's Modification of Ham's F-12 Medium (ATCC) supplemented with $0.3 \%$ BSA (Sigma-Aldrich), penicillin/streptomycin (Invitrogen) plus $1 \mu \mathrm{g} / \mathrm{ml}$ of TPCK-treated trypsin and incubated at $37{ }^{\circ} \mathrm{C}$ with $5 \% \mathrm{CO}_{2}$.

The viruses encoding WT, G45R and AA/NS1s were used to infect WT or IFNAR ${ }^{\text {null }}$ Let1 cells grown in 6-well plates in triplicate at an MOI of 1 or 1 . The numbers of plaque forming units (pfu) in the inoculum were reexamined by plaque assay to confirm the equality of the virus in each inoculum (Additional file 4: Fig. S4). The infected cells were maintained in DMEM (Gibco, Invitrogen) supplemented with $0.3 \%$ BSA (Sigma), penicillin/ streptomycin (Gibco, Invitrogen) plus $0.1 \mu \mathrm{g} / \mathrm{ml}$ of TPCK trypsin (Sigma) and incubated at $37{ }^{\circ} \mathrm{C}$ with $5 \% \mathrm{CO}_{2}$. The cell supernatants were collected at indicated time points. The viruses collected at each time point were titrated by tissue culture infectious dose $\left(\mathrm{TCID}_{50}\right)$ assay in MDCK cells and calculated as previously described [43].

\section{Expression of NS1 RBD}

E. coli strain BL21 transformed with $\mathrm{pQE}$ plasmids containing the amino acid 1-73 of the RBD of PR8 NS1 including WT or mutants G45R and AA were grown in Luria broth with $100 \mathrm{mg} / \mathrm{ml}$ ampicillin (Invitrogen). Protein expression was induced with $0.2 \mathrm{mM}$ IPTG (Invitrogen) at $37{ }^{\circ} \mathrm{C}$ for $18 \mathrm{~h}$. The cells were then pelleted by centrifugation at $4000 \mathrm{rpm}$ at $4{ }^{\circ} \mathrm{C}$ for $20 \mathrm{~min}$. The cell pellets were mixed well in $30 \mu \mathrm{l}$ of lysis buffer $(1 \mathrm{M}$ Tris, $300 \mathrm{mM} \mathrm{NaCl}, 1$ \% Triton $\mathrm{X}$ and 1 mM EDTA, pH 8.0). Subsequently, lysozyme and PMSF were added into the mixture at the final concentrations of $0.25 \mathrm{mg} / \mathrm{ml}$ and $1 \mathrm{mM}$, respectively. The mixture was vortex-mixed and incubated on ice for $30 \mathrm{~min}$. The cells suspension was homogenized by sonication followed by centrifugation at $10,000 \mathrm{~g}$ at $4{ }^{\circ} \mathrm{C}$ for $20 \mathrm{~min}$ and the supernatant containing the soluble fraction of RBD NS1 was collected. The proteins were purified using Protino Ni-TED Resingravity-flow column (Macherey-Nagel) under native condition and were then concentrated in a $3 \mathrm{kDa}-\mathrm{Cen}$ trifugal filter units (Millipore). The presentation of His-
RBD NS1 proteins were examined by SDS-PAGE using $15 \%$ acrylamide gels. Western blot analysis was performed using anti-NS1 rabbit hyperimmune serum and goat anti-rabbit IgG-HRP (Millipore) as primary and secondary antibodies, respectively.

\section{dsRNA-protein pull down assay}

To generate dsRNA labeled with biotin, the biotinylated sense and antisense single-stranded (ss) RNA were in vitro transcribed. Briefly, the dsDNA template was produced by amplification of a 72 bp DNA fragment using forward and reverse primers containing the promoter sequences for the binding of T7 and T3 RNA polymerases, respectively [44]. The dsDNA fragment was purified using a DNA purification kit (Qiagen) and used as the template to produce biotinylated ssRNA by in vitro transcription using the Biotin RNA Labeling Mix (Roche) plus T7 or T3 RNA polymerase (Promega). The remaining DNA in the in vitro transcription reaction was destroyed by using DNase I (Promega) and the biotinylated ssRNA was purified by using ethanol precipitation method. The purified biotinylated T7- and T3ssRNA were annealed at room temperature to obtain the biotinylated dsRNA and kept on ice until used in the binding reaction.

To optimize the pull down reaction, various concentrations of biotinylated dsRNA and NS1 RBD were examined in a checker board titration. Additional file 5: Fig. S5 shows an example of the titration results. The optimal condition was selected. In the pull down assay, $0.4 \mathrm{mg}$ of each purified NS1 was incubated with $0.6 \mu \mathrm{M}$ biotinylated dsRNA in $20 \mu \mathrm{l}$ of binding buffer containing $50 \mathrm{mM}$ Tris- $\mathrm{HCl}, \mathrm{pH}$ 8.3, $75 \mathrm{mM} \mathrm{KCl}, 3 \mathrm{mM} \mathrm{MgCl}$, $8 \%$ (v/v) glycerol, $0.1 \%$ (v/v) Triton X-100 (Amresco), $1 \mathrm{mM}$ DTT (Invitrogen), $5 \mu \mathrm{g}$ yeast tRNA (Roche) and 40 Unit RNase OUT (Invitrogen). The reaction mixtures were incubated at room temperature without agitation for $30 \mathrm{~min}$. After incubation, the dsRNA-NS1 complex was pulled down with NeutraAvidinAgarose Resin (Pierce, Thermo Scientific) by incubation at room temperature for $45 \mathrm{~min}$. The resin was boiled in Lamelli sample buffer (Bio-Rad). The pulled-down protein was separated by using SDS-PAGE in $15 \%$ gel. Western blot was performed by using anti-NS1 rabbit serum and goat-anti rabbit IgG-HRP (Millipore) as primary and secondary antibodies, respectively. The membrane was incubated in Clarity western ECL substrate (Bio-Rad) for $5 \mathrm{~min}$. The chemiluminescent signal was detected by $\mathrm{Fu}$ sion FX imaging analyzer (VilberLourmat).

\section{RIG-I mediated IFN $\beta$-promoter activity assay}

The plasmids expressing IFN $\beta$-promoter $(-125)$ firefly luciferase, Renilla Luciferase vector (pRL-TK; Promega), RIG-I and each PR8 NS1 (WT, G45R and AA) were co- 
transfected into $293 \mathrm{~T}$ cells grown in 24-well plate as previously described [45] with some modifications. Briefly, the indicated plasmids were transfected to the cells using XtremeGene HP transfection reagent (Roche). At $24 \mathrm{~h}$ post transfection (pt), the cells were lysed in passive lysis buffer (Invitrogen) and subjected for luciferase activity measurement using dual-luciferase kit following the manufacturer's instruction (Promega). The firefly luciferase activity signals were normalized to the Renilla luciferase activity. The experiments were done in triplicate. NS1 expression levels in transfected cells were shown by western blot analysis (Additional file 2: Fig. S2).

\section{Quantitative real-time PCR (qRT-PCR)}

Total RNA was isolated from A549 cells infected with the rX31 viruses encoding PR8 NS1 (WT and G45R) using Trizol reagent (Invitrogen) following the manufacturer's instruction. Total RNA was isolated by using Direct-zol ${ }^{\text {mix }}$ RNA MiniPrep (Zymo research). One microgram of total RNA was reverse transcribed using a high-capacity cDNA reverse transcription kit (Applied Biosystem). Realtime PCR was performed by using a 7500 Fast real-time PCR system (Applied Biosystem). In $15 \mu \mathrm{l}$ reaction, $0.4 \mathrm{mM}$ of forward and reverse primers and equal amount of cDNA were mixed with the SYBR Select master mix (Applied Biosystem) according to the manufacturer's protocol. The qRT- PCR cycle were conducted under the following conditions: enzyme activation at $95{ }^{\circ} \mathrm{C}$ for 2 min followed by 40 cycles of denaturation at $95{ }^{\circ} \mathrm{C}$ for $3 \mathrm{~s}$, annealing and extension at $60{ }^{\circ} \mathrm{C}$ for $30 \mathrm{~s}$. Three biological replicates and two technical repeats were performed for each sample. The housekeeping gene, $\beta$-actin, was used as a reference control. The data of each sample and negative control (mock infected cells) were normalized to the reference using the threshold cycle $\left(2^{-\Delta \Delta C T}\right)$ method [46].

\section{Data analysis}

Mean titers of the viruses at each time point, luciferase activity and relative mRNA expression level were compared and statistically calculated using analysis of variance (ANOVA) and student $t$-test method. Mean titers of the viruses as $\mathrm{TCID}_{50} / \mathrm{ml}$ was converted into base- 10 logarithms and plotted against time points and a bar corresponding to SEM was demonstrated. A difference was considered significant if the $p$-value was $<0.05$.

\section{Additional files}

Additional file 1: Fig. S1. Three replicates of dsRNA-RBD pulled down assay. $0.6 \mu \mathrm{M}$ of biotinylated-dsRNA and $0.4 \mathrm{mg}$ NS1-RBDs were used in the pulled down assay. The assay was performed using similar condition in three separated reactions. The pulled-down NS1-RBD protein was electrophoresed through $15 \%$ acrylamide gel and blotted onto a nitrocellulose membrane. Western blot was performed by using anti-NS1 rabbit serum and goat-anti rabbit lgG-HRP as primary and secondary antibodies, respectively. The protein was detected by chemiluminescent method. The G45R bound to dsRNA with lower capacity than the wild type (WT). The R38AK41A (AA) served as a negative control failed to bind with dsRNA. (TIFF $305 \mathrm{~kb}$ )

Additional file 2: Fig. S2. NS1 expression in transfected 293 T cells as examined by western blot analysis. At $24 \mathrm{~h}$ post transfection, the cells were lysed in passive lysis buffer (Invitrogen) and subjected for western blot analysis to measure NS1 levels. The lysates were mixed in Laemmli sample buffer (Bio-Rad) and denatured at $95^{\circ} \mathrm{C}$ for 5 mins prior to perform SDS-PAGE gel electrophoresis. Immunoblots were probed for NS1 protein (polyclonal rabbit anti-NS1; Thermoscientific) and $\beta$-actin (rabbit anti-actin antibody; Thermoscientific). Antibodies were detected by incubation with goat anti-rabbit HRP-linked antibody (Millipore). Lane $1=$ empty plasmid; Lanes 2 and $3=W T$ and G45R at $0.4 \mu$; Lanes 4 and $5=W T$ and G45R at $0.2 \mu \mathrm{g}$. (TIF $105 \mathrm{~kb}$ )

Additional file 3: Fig. S3. Western blot analysis of STAT1, pSTAT1 and NS1 protein. A549 cells were infected with rX31 encoding WT and G45R/ NS1 viruses at an MOI of 2. At $8 \mathrm{~h}$ post infection, cell lysates were clarified by centrifugation at 13,000 rpm for $15 \mathrm{~min}$. The lysates were mixed in Laemmli sample buffer (Bio-Rad) and denatured at $95^{\circ} \mathrm{C}$ for 5 mins prior to perform SDS-PAGE gel electrophoresis. Immunoblots were probed for STAT1 (purified rabbit anti-Stat1 N-terminus, BD Transduction Laboratories), pSTAT1 (purified mouse anti-Stat1 pY701 BD Transduction Laboratories), NS1 (polyclonal rabbit anti-NS1; Thermoscientific) and actin (purified mouse anti-actin Ab-5; BD Transduction Laboratories). Antibodies were detected by incubation with goat anti-rabbit (GE healthcare) or goat anti-mouse HRP-linked antibody (Cell signaling). The immunoblots were visualized by using ChemiDoc XRS imager (Bio-Rad). Band intensity of proteins was quantified by using Image Lab version 5.0 (Bio-Rad). (TIFF $2319 \mathrm{~kb}$ )

Additional file 4: Fig. S4. Plaque assay of the virus inoculums. The virus inoculums (WT and G45R/NS1) at MOI of 0.01 were serially diluted before they were infected onto MDCK cells to confirm that each inoculum had equal amount of the virus. Plaque assay of the viruses at $10^{-5}$ dilution is shown. The average virus titers of both WT and G45R/NS1 inoculums are $5 \times 10^{6} \mathrm{pfu} / \mathrm{ml}$. (TIFF $1781 \mathrm{~kb}$ )

Additional file 5: Fig. S5. Checker board titration of dsRNA and NS1-RBD. Different concentrations of biotinylated-dsRNA and NS1-RBD were titrated in the dsRNA-protein pulled down assay. The pulled-down NS1-RBD protein was separated by SDS-PAGE in $15 \%$ acrylamide gel and blotted onto a nitrocellulose membrane. Western blot was performed by using anti-NS1 rabbit serum and goat-anti rabbit lgG-HRP as primary and secondary antibodies, respectively. The protein was detected by chemiluminescent method. Lanes 1,2 and 3 are the reactions containing $0.4 \mu \mathrm{M}$ dsRNA and 0.2, 0.4 and $0.6 \mathrm{mg}$ NS1-RBDs. Lanes 4, 5 and 6 are the reactions containing $0.6 \mu \mathrm{M}$ dsRNA and $0.2,0.4$ and $0.6 \mathrm{mg} \mathrm{NS1-}$ RBDs, respectively. $\mathrm{N} 1$ and $\mathrm{N} 2$ are the negative control reactions without dsRNA or RBD, respectively. (TIFF $204 \mathrm{~kb}$ )

\section{Acknowledgements}

This work was supported by Thailand Research Fund (TRF) through the Royal Golden Jubilee Ph.D. Program and Kasetsart University (Grant No. PHD/0153/2552), National Research Council of Thailand (NRCT) (Grant No. Po Ko/2554-36) and the American Lebanese Syrian Associated Charities (ALSAC).

\section{Authors' contribution}

CK designed and performed the experiments and drafted the manuscript. MZ carried out the plaque assay and drafted the manuscript. HH and RW participated in the study design and revised the manuscript. PL supervised the project and revised the manuscript. All authors read and approved the final manuscript.

\section{Competing interests}

The authors declare that they have no competing interests.

\section{Author details}

IInterdisciplinary Graduate Program in Genetic Engineering, The Graduate School, Kasetsart University, Bangkok 10900, Thailand. ${ }^{2}$ Department of 
Infectious Diseases, Division of Virology, St. Jude Children's Research Hospital, Memphis 38105-2794, TN, USA. ${ }^{3}$ Department of Infectious Diseases, St. Jude Children's Research Hospital, Memphis 38105-2794, TN, USA. ${ }^{4}$ Department of Microbiology and Immunology, Faculty of Veterinary Medicine, Kasetsart University, 50th Ngamwongwan Rd., Chatuchak, Bangkok 10900, Thailand. ${ }^{5}$ Center for Advances Studies in Agriculture and Food, KU Institute for Advanced Studies, Kasetsart University, Bangkok 10900, Thailand.

\section{Received: 21 January 2016 Accepted: 6 July 2016}

Published online: 12 July 2016

\section{References}

1. Liu Q, Zhou B, Ma W, Bawa B, Ma J, Wang W, et al. Analysis of recombinant H7N9 wild-type and mutant viruses in pigs shows that the Q226L mutation in HA is important for transmission. J Virol. 2014;88:8153-65.

2. Zhang $Y$, Zhang Q, Gao Y, He X, Kong H, Jiang Y, et al. Key molecular factors in hemagglutinin and PB2 contribute to efficient transmission of the 2009 H1N1 pandemic influenza virus. J Virol. 2012;86:9666-74.

3. Mok CK, Lee HH, Lestra M, Nicholls JM, Chan MC, Sia SF, et al. Amino acid substitutions in polymerase basic protein 2 gene contribute to the pathogenicity of the novel a/H7N9 influenza virus in mammalian hosts. J Virol. 2014;88:3568-76.

4. Garcia-Sastre A. Induction and evasion of type I interferon responses by influenza viruses. Virus Res. 2011;162:12-8.

5. Takaoka A, Yanai H. Interferon signalling network in innate defence. Cell Microbiol. 2006:8:907-22

6. Opitz B, Rejaibi A, Dauber B, Eckhard J, Vinzing M, Schmeck B, et al. IFNbeta induction by influenza a virus is mediated by RIG-I which is regulated by the viral NS1 protein. Cell Microbiol. 2007;9:930-8.

7. Davis WG, Bowzard JB, Sharma SD, Wiens ME, Ranjan P, Gangappa S, et al. The $3^{\prime}$ untranslated regions of influenza genomic sequences Are 5'PPPindependent ligands for RIG-I. PLoS One. 2012;7, e32661.

8. Lee N, Wong CK, Hui DS, Lee SK, Wong RY, Ngai KL, et al. Role of human toll-like receptors in naturally occurring influenza a infections. Influenza Other Respir Viruses. 2013;7:666-75.

9. Gauzzi MC, Velazquez L, Mckendry R, Mogensen KE, Fellous M, Pellegrini S. Interferon-alpha-dependent activation of Tyk2 requires phosphorylation of positive regulatory tyrosines by another kinase. J Biol Chem. 1996;271: 20494-500.

10. Garcia MA, Gil J, Ventoso I, Guerra S, Domingo E, Rivas C, et al. Impact of protein kinase PKR in cell biology: from antiviral to antiproliferative action. Microbiol Mol Biol Rev. 2006;70:1032-60.

11. Au-Yeung N, Mandhana R, Horvath CM. Transcriptional regulation by STAT1 and STAT2 in the interferon JAK-STAT pathway. JAKSTAT. 2013;2, e23931.

12. Haye K, Burmakina S, Moran T, Garcia-Sastre A, Fernandez-Sesma A. The NS1 protein of a human influenza virus inhibits type I interferon production and the induction of antiviral responses in primary human dendritic and respiratory epithelial cells. J Virol. 2009;83:6849-62.

13. Donelan NR, Basler CF, Garcia-Sastre A. A recombinant influenza a virus expressing an RNA-binding-defective NS1 protein induces high levels of beta interferon and is attenuated in mice. J Virol. 2003;77:13257-66.

14. Garcia-Sastre A, Egorov A, Matassov D, Brandt S, Levy DE, Durbin JE, et al. Influenza a virus lacking the NS1 gene replicates in interferon-deficient systems. Virology. 1998;252:324-30.

15. Kochs G, Garcia-Sastre A, Martinez-Sobrido L. Multiple anti-interferon actions of the influenza a virus NS1 protein. J Virol. 2007:81:7011-21.

16. Mibayashi M, Martinez-Sobrido L, Loo YM, Cardenas WB, Gale Jr M, GarciaSastre A. Inhibition of retinoic acid-inducible gene I-mediated induction of beta interferon by the NS1 protein of influenza a virus. J Virol. 2007;81:514-24.

17. Min JY, Krug RM. The primary function of RNA binding by the influenza a virus NS1 protein in infected cells: inhibiting the 2'-5' oligo (a) synthetase/ RNase L pathway. Proc Natl Acad Sci U S A. 2006;103:7100-5.

18. Min JY, Li S, Sen GC, Krug RM. A site on the influenza a virus NS1 protein mediates both inhibition of PKR activation and temporal regulation of viral RNA synthesis. Virology. 2007:363:236-43.

19. Li S, Min JY, Krug RM, Sen GC. Binding of the influenza a virus NS1 protein to PKR mediates the inhibition of its activation by either PACT or doublestranded RNA. Virology. 2006;349:13-21.

20. Lekcharoensuk P, Wiriyarat W, Petcharat N, Lekcharoensuk C, Auewarakul P, Richt JA. Cloned cDNA of a/swine/lowa/15/1930 internal genes as a candidate backbone for reverse genetics vaccine against influenza a viruses. Vaccine. 2012;30:1453-9.

21. Kaewborisuth C, Tungtumniyom N, Phecharat N, Lekcharoensuk C, Lekcharoensuk P. NS gene of influenza virus a/swine/IA/15/30 increases the replication rate of a/Puerto Rico/8/34 in MDCK and Vero cells. Thai J Vet Med. 2015:45:9.

22. Hoffmann E, Neumann G, Kawaoka Y, Hobom G, Webster RG. A DNA transfection system for generation of influenza a virus from eight plasmids. Proc Natl Acad Sci U S A. 2000;97:6108-13.

23. Murakami S, Horimoto T, le Mai Q, Nidom CA, Chen H, Muramoto Y, et al. Growth determinants for H5N1 influenza vaccine seed viruses in MDCK cells. J Virol. 2008;82:10502-9.

24. Pichlmair A, Schulz O, Tan CP, Naslund TI, Liljestrom P, Weber F. Reis e Sousa C: RIG-I-mediated antiviral responses to single-stranded RNA bearing 5'-phosphates. Science. 2006:314:997-1001.

25. Yoneyama M, Kikuchi M, Natsukawa T, Shinobu N, Imaizumi T, Miyagishi M, et al. The RNA helicase RIG-I has an essential function in double-stranded RNA-induced innate antiviral responses. Nat Immunol. 2004;5:730-7.

26. Rehwinkel J, Tan CP, Goubau D, Schulz O, Pichlmair A, Bier K, et al. Reis e Sousa C: RIG-I detects viral genomic RNA during negative-strand RNA virus infection. Cell. 2010;140:397-408.

27. Rosenberger CM, Podyminogin RL, Askovich PS, Navarro G, Kaiser SM, Sanders CJ, et al. Characterization of innate responses to influenza virus infection in a novel lung type I epithelial cell model. J Gen Virol. 2014;95:350-62.

28. Hale BG, Randall RE, Ortin J, Jackson D. The multifunctional NS1 protein of influenza a viruses. J Gen Virol. 2008;89:2359-76.

29. Lu Y, Wambach M, Katze MG, Krug RM. Binding of the influenza virus NS1 protein to double-stranded RNA inhibits the activation of the protein kinase that phosphorylates the elF-2 translation initiation factor. Virology. 1995;214:222-8

30. Mi Z, Ma Y, Tong Y. Avian influenza virus H5N1 induces rapid interferonbeta production but shows more potent inhibition to retinoic acidinducible gene I expression than H1N1 in vitro. Virol J. 2012;9:145

31. Solorzano A, Webby RJ, Lager KM, Janke BH, Garcia-Sastre A, Richt JA Mutations in the NS1 protein of swine influenza virus impair anti-interferon activity and confer attenuation in pigs. J Virol. 2005:79:7535-43.

32. Rajsbaum R, Albrecht RA, Wang MK, Maharaj NP, Versteeg GA, Nistal-Villan E, et al. Species-specific inhibition of RIG-I ubiquitination and IFN induction by the influenza a virus NS1 protein. PLoS Pathog. 2012;8, e1003059.

33. Ramos I, Carnero E, Bernal-Rubio D, Seibert CW, Westera L, Garcia-Sastre A et al. Contribution of double-stranded RNA and CPSF30 binding domains of influenza virus NS1 to the inhibition of type I interferon production and activation of human dendritic cells. J Virol. 2013:87:2430-40.

34. Salahuddin P, Khan AU. Structural and functional analysis of NS1 and NS2 proteins of H1N1 subtype. Genomics Proteomics Bioinformatics. 2010;8:190-9.

35. Guo Z, Chen L-m, Zeng H, Gomez JA, Plowden J, Fujita T, et al. NS1 protein of influenza a virus inhibits the function of intracytoplasmic pathogen sensor, RIG-I. Am J Respir Cell Mol Biol. 2007;36:263-9.

36. Gack MU, Albrecht RA, Urano T, Inn KS, Huang IC, Carnero E, et al. Influenza a virus NS1 targets the ubiquitin ligase TRIM25 to evade recognition by the host viral RNA sensor RIG-I. Cell Host Microbe. 2009:5:439-49.

37. Veckman V, Österlund P, Fagerlund R, Melén K, Matikainen S, Julkunen I. TNF- $a$ and IFN- $a$ enhance influenza-a-virus-induced chemokine gene expression in human A549 lung epithelial cells. Virology. 2006;345:96-104.

38. Davidson S, Crotta S, McCabe TM, Wack A: Pathogenic potential of interferon a $\beta$ in acute influenza infection. Nat Commun. 2014;5:3864. doi:10.1038/ncomms4864.

39. Spesock A, Malur M, Hossain MJ, Chen LM, Njaa BL, Davis CT, et al. The virulence of 1997 H5N1 influenza viruses in the mouse model is increased by correcting a defect in their NS1 proteins. J Virol. 2011;85:7048-58.

40. Dankar SK, Wang S, Ping J, Forbes NE, Keleta L, Li Y, et al. Influenza a virus NS1 gene mutations F103L and M106I increase replication and virulence. Virol J. 2011:8:13.

41. Patel JR, Vora KP, Tripathi S, Zeng H, Tumpey TM, Katz JM, et al. Infection of lung epithelial cells with pandemic 2009 a(H1N1) influenza viruses reveals isolate-specific differences in infectivity and host cellular responses. Viral Immunol. 2011;24:89-99.

42. Hoffmann E, Krauss S, Perez D, Webby R, Webster RG. Eight-plasmid system for rapid generation of influenza virus vaccines. Vaccine. 2002;20:3165-70.

43. Reed $\amalg$, Muench $\mathrm{H}$. A simple method of estimating fifty percent endpoints. Am J Epidemiol. 1938;27:493-7. 
44. Klaywong K, Khutrakul G, Choowongkomon K, Lekcharoensuk C, Petcharat $\mathrm{N}$, Leckcharoensuk $\mathrm{P}$, et al. Screening for lead compounds and herbal extracts with potential anti-influenza viral activity. Southeast Asian J Trop Med Public Health. 2014:45:62-74.

45. Tawaratsumida K, Phan V, Hrincius ER, High AA, Webby R, Redecke V, et al. Quantitative proteomic analysis of the influenza a virus nonstructural proteins NS1 and NS2 during natural cell infection identifies PACT as an NS1 target protein and antiviral host factor. J Virol. 2014;88:9038-48.

46. Livak KJ, Schmittgen TD. Analysis of relative gene expression data using real-time quantitative $P C R$ and the $2(-$ delta delta $C(T))$ method. Methods. 2001;25:402-8

Submit your next manuscript to BioMed Central and we will help you at every step:

- We accept pre-submission inquiries

- Our selector tool helps you to find the most relevant journal

- We provide round the clock customer support

- Convenient online submission

- Thorough peer review

- Inclusion in PubMed and all major indexing services

- Maximum visibility for your research

Submit your manuscript at www.biomedcentral.com/submit 